\title{
The Role of Multiparametric Magnetic Resonance Imaging to Detect the Extracapsular Extension in Robot- Assisted Radical Prostatectomy
}

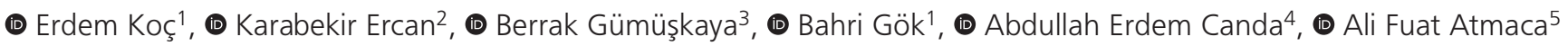 \\ ${ }^{1}$ Ankara Yıldırım Beyazıt University Faculty of Medicine, Ankara State Hospital, Clinic of Urology, Ankara, Turkey \\ ${ }^{2}$ Ankara State Hospital, Clinic of Radiology, Ankara, Turkey \\ ${ }^{3}$ Ankara Yıldırım Beyazıt University Faculty of Medicine, Ankara State Hospital, Clinic of Pathology, Ankara, Turkey \\ ${ }^{4}$ Koç University Faculty of Medicine, Department of Urology, Istanbul, Turkey \\ ${ }^{5}$ Ankara Memorial Hospital, Clinic of Urology, Ankara, Turkey
}

\begin{abstract}
Objective: This study aimed to evaluate the effectiveness of multiparametric prostate magnetic resonance imaging (mpMRI) in detecting extracapsular extension (ECE) and its impact on our preoperative surgical plan.

Materials and Methods: Overall, 150 patients who had undergone preoperative mpMRI and robot-assisted laparoscopic radical prostatectomy (RALRP) between June 2016 and March 2018 were enrolled. The preoperative International index of Erectile Function (IIEF) score of the patients was 22 or higher. On initial assessment, the patients' prostate specific antigen levels and digital rectal examination and pathology results of prostate biopsy specimens were evaluated to determine whether neurovascular bundle (NVB)-sparing surgery is feasible and appropriate. On the second evaluation, mpMRI results were considered in addition to the parameters included during the first evaluation to decide whether NVB-sparing surgery should be performed.

Results: The mean age of the patients was 65.2 years. The sensitivity, specificity, positive predictive value and negative predictive value of mpMRI were $89.6 \%$, $90.2 \%, 81.1 \%$ and $94.8 \%$, respectively. According to mpMRI findings, the surgical plan at the initial evaluation changed in 35 (23.3\%) of the cases.

Conclusion: The high rate of detection of ECE with mpMRI prior to RALRP may guide the surgeon to decide for NVB-sparing surgery.

Keywords: Prostate cancer, multiparametric magnetic resonance imaging, robotic surgery, radical prostatectomy
\end{abstract}

\section{Introduction}

Pathological extracapsular extension (ECE) after radical prostatectomy increases the stage of the disease and cancerrelated mortality $(1,2)$. ECE may be detected by palpation during open surgery; however, it is difficult to detect ECE during the course of robotic surgery due to the absence of tactile sensation. The combination of transrectal ultrasonography and digital rectal examination (DRE) is not sufficient for the accurate detection of ECE (3). Nomograms such as Partin and Memorial Sloan Kettering Cancer Center provide information on ECE; however, they do not reveal detailed anatomical information
$(4,5)$. Accurately localising the tumour is beneficial in performing neurovascular bundle (NVB)-sparing surgery by preventing excessive extraprostatic tissue resection, determining the margins of the tissue which needed to be removed in order to achieve negative surgical margins which may provide better functional and oncological results $(6,7)$. In recent years, multiparametric prostate magnetic resonance imaging (mpMRI) has been the most promising technique in detecting and staging of prostate cancer, which can present cite-specific results in $\operatorname{ECE}(8,9)$. In this study, we aimed to evaluate the effectiveness of mpMRI in detecting ECE and whether it affected our preoperative surgical plan.

Cite this article as: Koç E, Ercan K, Gümüşkaya B, Gök B, Canda AE, Atmaca AF. The Role of Multiparametric Magnetic Resonance Imaging to Detect the Extracapsular Extension in Robot-Assisted Radical Prostatectomy. Bull Urooncol 2020;19(4):206-209

Address for Correspondence: Erdem Koç, Ankara Yıldırım Beyazıt University Faculty of Medicine, Ankara State Hospital, Clinic of Urology, Ankara, Turkey Phone: +90 5066614366 E-mail: drerdemkoc@gmail.com ORCID-ID: orcid.org/0000-0003-3439-2105 


\section{Materials and Methods}

The data of 150 patients who underwent mpMRI before robotassisted laparoscopic radical prostatectomy (RALRP) between June 2016 and March 2018 and whose IIEF score was >21 were retrospectively reviewed. mpMRI was performed using a 3-Tesla Siemens system without an endorectal coil. Diffusionweighted MRI, T2-weighted MRI and dynamic contrastenhanced MRI techniques were used for the localisation of the tumour site and possible ECE. mpMRI was performed according to the 2012 European Society of Urogenital Radiology prostate MRI guidelines (10) by a single radiologist (KE) with a 10year experience; the radiologist was not blinded of the clinical diagnoses, DRE findings, prostate specific antigen (PSA) levels and biopsy results of the patients.

Moreover, RALRP was performed by two experienced surgeons (AFA, AEC). In the preoperative evaluation, the surgery planning was performed in two steps. First, DRE findings, PSA level and biopsy results were considered to determine whether NVB should be preserved or not. The final decision for propriety of NVB-sparing was made according to the mpMRI findings.

Patients who had previously received hormone therapy or radiotherapy and those whose mpMRI was reported in another centre were excluded from the study.

All prostatectomy specimens were examined by one dedicated uropathologist. The macroscopically dyed, formalin-fixed specimens were serially sectioned within a 3-mm-thick cutting surface, from apex to base and perpendicular to the posterior surface. Slices were further cut into 3-5 $\mu \mathrm{m}$ microscopic pieces and then stained with hematoxylin and eosin stain. ECE was defined as the tumour extending beyond the prostate capsule, through the extraprostatic tissues. TNM classification was used to determine the pathological T (pT) stage. Our study was approved by the Ankara Yıldırım Beyazıt University Ethics Committee (approval no: 16, date: 22.04.2020). Clinical data were retrospectively reviewed and analysed.

\section{Statistical Analysis}

Data analysis was performed using the IBM SPSS Statistics version 20.0 software (IBM Corporation, Armonk, NY, USA). Whether the continuous variables were distributed normally was determined by Kolmogorov-Smirnov test. Mann-Whitney $U$ test was used for not normally distributed continuous variables, and the results were presented as median (minimum - maximum). The number of cases (n) and percentages (\%) were used for categorical data. Categorical variables were analysed by continuity corrected chisquare test or Fisher's Exact test. Additionally, the diagnostic indicators (i.e., sensitivity, specificity, positive predictive value (PPV) and negative predictive value (NPV) and accuracy) for MRI were calculated. The statistical significance of the diagnostic value of mpMRI according to the gold standard pathology in terms of determining ECE was evaluated. P-value $<0.05$ was considered statistically significant.

\section{Results}

Overall, 177 patients who underwent RALRP were analysed. We excluded 1 patient who was receiving hormonotherapy, 4 patients whose mpMRI tests were taken in another health centre, and 22 patients who has IIEF score $<22$. Finally, 150 patients were included. The median age of the patients was 65 years (49-75). The demographic characteristics and clinical and pathological

\begin{tabular}{|c|c|}
\hline Variables & $n=150$ \\
\hline Age (years) Median (min-max) & $65(49-75)$ \\
\hline Preoperative PSA value $(\mathrm{ng} / \mathrm{mL}$ ) Median (min-max) & $7(1.2-96)$ \\
\hline Prostate weight (g) Median (min-max) & $40(15-286)$ \\
\hline Time from mpMRI to surgery (days) Median (min-max) & $46(2-359)$ \\
\hline Time from biopsy to surgery (days) Median (min-max) & $101(53-475)$ \\
\hline Time from mpMRI to biopsy (days) Median (min-max) & $55(40-426)$ \\
\hline Largest lesion size in mpMRI (mm) Median (min-max) & $20(5-45)$ \\
\hline ECE positivity in mpMRI $n(\%)$ & $53(35.3 \%)$ \\
\hline \multicolumn{2}{|l|}{ PIRADS score n (\%) } \\
\hline 3 & $9(17.0 \%)$ \\
\hline 4 & $22(41.5 \%)$ \\
\hline 5 & $22(41.5 \%)$ \\
\hline \multicolumn{2}{|l|}{ Clinical stage $\mathrm{n}(\%)$} \\
\hline T1c & $86(57.3 \%)$ \\
\hline $\mathrm{T} 2 \mathrm{a}$ & $41(27.3 \%)$ \\
\hline $\mathrm{T} 2 \mathrm{~b}$ & $11(7.3 \%)$ \\
\hline $\mathrm{T} 2 \mathrm{c}$ & $9(6.0 \%)$ \\
\hline T3 & $3(2 \%)$ \\
\hline Positive core number in biopsy Median (min-max) & $3(1-10)$ \\
\hline \multicolumn{2}{|l|}{ Biopsy Gleason score n (\%) } \\
\hline Grade Group 1 & $78(52.0 \%)$ \\
\hline Grade Group 2 & $40(26.7 \%)$ \\
\hline Grade Group 3 & $11(7.3 \%)$ \\
\hline Grade Group 4 & $14(9.3 \%)$ \\
\hline Grade Group 5 & $7(4.7 \%)$ \\
\hline \multicolumn{2}{|l|}{ Gleason score in specimen $\mathrm{n}(\%)$} \\
\hline Grade Group 1 & $62(41.3 \%)$ \\
\hline Grade Group 2 & $47(31.3 \%)$ \\
\hline Grade Group 3 & $23(15.3 \%)$ \\
\hline Grade Group 4 & $10(6.7 \%)$ \\
\hline Grade Group 5 & $8(5.3 \%)$ \\
\hline Cancer percentage in specimen Median (min-max) & $10(1-90)$ \\
\hline Tumor multifocality in specimen $\mathrm{n}(\%)$ & $50(33.3 \%)$ \\
\hline PSM n (\%) & $16(10.7 \%)$ \\
\hline ECE in pathology $n(\%)$ & $48(32.0 \%)$ \\
\hline \multicolumn{2}{|l|}{ LN dissection $\mathrm{n}(\%)$} \\
\hline Not performed & $78(52.0 \%)$ \\
\hline Benign & $66(44.0 \%)$ \\
\hline Malign & $6(4.0 \%)$ \\
\hline \multicolumn{2}{|l|}{ Pathologic T stage n (\%) } \\
\hline $\mathrm{T} 2 \mathrm{a}$ & $31(20.7 \%)$ \\
\hline
\end{tabular}




\begin{tabular}{|l|l|}
\hline \multicolumn{2}{|l|}{ table 1 continuation } \\
\hline T2b & $4(2.7 \%)$ \\
\hline T2c & $64(42.6 \%)$ \\
\hline T3a & $44(29.3 \%)$ \\
\hline T3b & $7(4.7 \%)$ \\
\hline NVB sparing n (\%) & $23(15.3 \%)$ \\
\hline Not performed & $26(17.4 \%)$ \\
\hline Unilaterally performed & $101(67.3 \%)$ \\
\hline Bilaterally performed & $\begin{array}{l}\text { PSM: Positive surgical margin, LN: Lymph node, NVB: Neurovascular bundle, } \\
\text { ECE: Extracapsular extension, PSA: Prostate specific antigen, NVB: Neurovascular } \\
\text { bundle, LN: Lymph node, mpMRI: Multiparametric magnetic resonance } \\
\text { imaging, ECE: Extracapsular extension, PIRADS: Prostate imaging reporting and } \\
\text { data system }\end{array}$ \\
\hline
\end{tabular}

results of the patients are smmarized in Table 1. mpMRI result was reported as ECE in 53 patients. ECE was detected in 48 patients in the final pathology after radical prostatectomy. NVBsparing surgery was performed in 127 (84.7\%) patients.

The initial surgical plan changed in $35(23.3 \%)$ of the cases according to $\mathrm{mpMRI}$ findings reported during the preoperative evaluation. In 21 (60\%) of these 35 patients, the surgical plan was changed from non-NVB-sparing surgery to NVB-sparing surgery. In the remaining 14 (40\%) patients, the surgical plan was changed from NVB-sparing surgery to non-NVB-sparing, considering the mpMRI results.

When the patients were divided into two groups according to final pathology results as ECE detected $(n=48)$ or not detected $(n=102)$, there was no statistically significant difference between the two groups in terms of age $(p=0.951)$. The median cancer percentage, median prostate imaging reporting and data system score and the largest lesion size in mpMRI were significantly higher in the group with ECE than in the group without ECE $(\mathrm{p}<0.001)$ (Table 2).

The sensitivity, specificity, PPV and NPV of mpMRI for detection of ECE were $89.6 \%, 90.2 \%, 81.1 \%$ and $94.8 \%$, respectively. The diagnostic accuracy rate of mpMRI for ECE was $90 \%$.

\section{Discussion}

In this current study, mpMRI was shown as a convenient method with high sensitivity (89.6\%) and specificity (90.2\%) in preoperative evaluation for ECE. Our surgical plan which we determined through the routine examination before the surgery was changed in the preoperative period in a quarter of the patients through the mpMRI results.

In a study conducted by Feng et al. (11), which included 112 patients, the sensitivity, specificity, PPV and NPV of mpMRI for ECE were $84.6 \%, 87.2 \%, 66.7 \%$ and $94.9 \%$, respectively. However, this study did not mention whether the radiologist knew about the clinical characteristics and biopsy pathology results of the patients. Moreover, in a study by Gaunay et al. (12) with 74 patients, the sensitivity, specificity, PPV, NPV and overall accuracy rate of mpMRI in detecting ECE were $58.3 \%$, $97.8 \%, 93.3 \%, 81.5 \%$ and $84.1 \%$, respectively. However, they did not state the experience of the radiologist and whether the radiologist had information about the patients' data. Park et al.

\begin{tabular}{|c|c|c|c|}
\hline Variables & $\begin{array}{l}\text { ECE positive } \\
(\mathrm{n}=48)\end{array}$ & $\begin{array}{l}\text { ECE negative } \\
(n=102)\end{array}$ & p-value \\
\hline $\begin{array}{l}\text { Age (years) Median } \\
\text { (min-max) }\end{array}$ & $64.5(51-75)$ & $65(49-75)$ & $0.966 \dagger$ \\
\hline $\begin{array}{l}\text { Cancer percentage } \\
\text { in specimen } \\
\text { Median (min-max) }\end{array}$ & $7(1-53)$ & $23.5(4-90)$ & $<0.001 \dagger^{*}$ \\
\hline $\begin{array}{l}\text { Largest lesion size in } \\
\text { mpMRI (mm) } \\
\text { Median (min-max) }\end{array}$ & $17(5-40)$ & $27.5(10-45)$ & $<0.001 \dagger^{*}$ \\
\hline $\begin{array}{l}\text { ECE positivity in } \\
\text { mpMRI } n(\%)\end{array}$ & $10(9.8 \%)$ & $43(89.6 \%)$ & $<0.001 \ddagger^{*}$ \\
\hline \multicolumn{3}{|l|}{ PIRADS score n (\%) } & $<0.001 \ddagger^{*}$ \\
\hline 3 & $5(50.0 \%)$ & $4(9.3 \%)$ & \\
\hline 4 & $5(50.0 \%)$ & $17(39.5 \%)$ & \\
\hline 5 & $0(0.0 \%)$ & $22(51.2 \%)$ & \\
\hline
\end{tabular}

(13) reported that the sensitivity, specificity, PPV, NPV and overall accuracy rate of mpMRI in predicting T3 prostate cancer were $55.9 \%, 82.2 \%, 59.1 \%, 80.2 \%$ and $73.9 \%$, respectively. In this study, even though the sensitivity of the mpMRI was low, it was significantly and relatively high in stage $\mathrm{T} 3$ disease. However, the radiologists who interpreted the mpMRI findings did not know the PSA values, biopsy pathology results and DRE of the patients. The difference in sensitivity of mpMRI in diagnosis of ECE through the different studies may depend on the patient selection, the technique differences in mpMRI imaging such as field strengths, sequence and coil types and may be affected by the experience and/or the knowledge of the radiologist (14). In the current study, factors such as the knowledge of the radiologist about the clinical findings, PSA levels and biopsy results of the patients may have contributed to the increased sensitivity and specificity findings of mpMRI in detecting ECE.

In radical prostatectomy, the main goal is to provide negative surgical margins and to perform NVB-sparing surgery. Partin and MSKS nomograms consider clinical stage, biopsy Gleason score and PSA values in preoperative detection of $\operatorname{ECE}(4,5)$. Even though they are widely used, their accuracy rate in determining ECE varies in different studies $(15,16)$. mpMRI provides surgeons detailed anatomical information useful for the establishment of a surgical plan. In this respect, mpMRI is an advantageous technique which provides information in both the detection and cite-specific localisation of the ECE. The absence of tactile sensation in robotic surgery makes it more valuable to use a sensitive and specific method such as mpMRI in addition to the routine examinations used. The surgeon pays more attention during the exploration of the area indicated as ECE in mpMRI to provide surgical margin negativity. Erectile dysfunction is one of the most common complications of RP. This crucial complication is observed less frequently in patients who underwent NVBsparing surgery than in those who had non-NVB-sparing surgery (17). Selective NVB-sparing surgery can be performed even in 
T3 disease to preserve erectile function (6). The absence of ECE in $\mathrm{mpMRI}$ and more desirable functional results may help the surgeon decide to perform NVB-sparing surgery.

It has been shown in previous studies that mpMRI contributed to the changes in preoperative plans in radical prostatectomy cases $(13,18,19,20,21)$. Park et al. (13) reported that mpMRI affected the surgical plan for $26 \%$ of the patients. In a study performed by McClure et al. (18), the surgical plan changed for 28 of 104 patients (27\%). Radtke et al. (21) reported a 31.1\% change in their initial surgical plan after the mpMRI results. In the current study, we consistently observed a $23.3 \%$ change in initial surgical plan due to mpMRI results. On the other hand, the high sensitivity and specificity of mpMRI in the current study may explain the higher rate of NVB-sparing and less surgical margin positivity compared to those in the literature $(20,22)$.

\section{Study Limitations}

The single-centre design, retrospective nature and evaluation of the mpMRI by a single experienced radiologist may be considered as the limitations of this study. All of the RALRP operations were performed by the same experienced surgeons, which may also be accepted as another limitation of this study.

\section{Conclusion}

mpMRI, which is performed during the preoperative period of the RALRP, appears to be a reliable technique in detecting the presence and localisation of the ECE, with high sensitivity and high specificity. Preoperative mpMRI may guide the surgeon in deciding to perform NVB preservation during RALRP.

\section{Acknowledgements}

Publication: This study was presented in $8^{\text {th }}$ Eurasian Urooncology Congress on June 28 - July 1 in 2018 Tbilisi, Georgia.

Contribution: There is not any contributors who may not be listed as authors.

Conflict of Interest: No conflict of interest was declared by the authors.

Financial Disclosure: The authors declared that this study received no financial support.

\section{Ethics}

Ethics Committee Approval: Our study was approved by the Ankara Yıldırım Beyazıt University Ethics Committee (approval no: 16 , date: 22.04 .2020 ).

Informed Consent: Clinical data were retrospectively reviewed and analysed.

Peer-review: Externally peer-reviewed.

\section{Authorship Contributions}

Surgical and Medical Practices: A.F.A., A.E.C., Concept: E.K., Design: E.K., Data Collection or Processing: E.K., B.G., K.E., B.G., Analysis or Interpretation: E.K., Literature Search: E.K., B.G., Writing: E.K.

\section{References}

1. Wheeler TM, Dillioglugil O, Kattan MW, et al. Clinical and pathological significance of the level and extent of capsular invasion in clinical stage T1-2 prostate cancer. Hum Pathol 1998;29:856-862.
2. Hull GW, Rabbani F, Abbas F, et al. Cancer control with radical prostatectomy alone in 1,000 consecutive patients. J Urol 2002; 167:528-534.

3. Hsu $\mathrm{CY}$, Joniau $\mathrm{S}$, Oyen $\mathrm{R}$, et al. Detection of clinical unilateral T3a prostate cancer- by digital rectal examination or transrectal ultrasonography? BJU Int 2006;98:982-985.

4. Partin AW, Kattan MW, Subong EN, et al. Combination of prostatespecific antigen, clinical stage, and gleason score to predict pathological stage of localized prostate cancer. A multi-institutional update. JAMA 1997;277:1445-1451.

5. Ohori M, Kattan MW, Koh $\mathrm{H}$, et al. Predicting the presence and side of extracapsular extension: a nomogram for staging prostate cancer. J Urol 2004;171:1844-1849.

6. Lightfoot AJ, Su YK, Sehgal SS, et al. Positive surgical margin trends in patients with pathologic T3 prostate cancer treated with robotassisted radical prostatectomy. J Endourol 2015;29:634-639.

7. Loeb S, Smith ND, Roehl KA, Catalona WJ. Intermediate-term potency, continence, and survival outcomes of radical prostatectomy for clinically high-risk or locally advanced prostate cancer. Urology 2007;69:1170-1175.

8. Sciarra A, Barentsz J, Bjartell A, et al. Advances in magnetic resonance imaging: how they are changing the management of prostate cancer. Eur Urol 2011;59:962-977.

9. Eberhardt SC, Carter S, Casalino DD, et al. ACR appropriateness criteria prostate cancer-pretreatment detection, staging, and surveillance. J Am Coll Radiol 2013;10:83-92.

10. Barentsz JO, Richenberg J, Clements R, et al. ESUR prostate MR guidelines 2012. Eur Radiol 2012;22:746-757.

11. Feng TS, Sharif-Afshar AR, Wu J, et al. Multiparametric MRI improves accuracy of clinical nomograms for predicting extracapsular extension of prostate cancer. Urology 2015;86:332-337.

12. Gaunay GS, Patel V, Shah P, et al. Multi-parametric MRI of the prostate: factors predicting extracapsular extension at the time of radical prostatectomy. Asian J Urol 2017;4:31-36.

13. Park $B H$, Jeon $H G$, Jeong $B C$, et al. Influence of magnetic resonance imaging in the decision to preserve or resect neurovascular bundles at robotic assisted laparoscopic radical prostatectomy. J Urol 2014; 192:82-88.

14. Stabile A, Giganti F, Rosenkrantz AB, et al. Multiparametric MRI for prostate cancer diagnosis: current status and future directions. Nat Rev Urol 2020;17:41-61.

15. Boesen L, Chabanova E, Løgager V, et al. Prostate cancer staging with extracapsular extension risk scoring using multiparametric MRI: a correlation with histopathology. Eur Radiol 2015;25:1776-1785.

16. Pinaquy JB, De Clermont-Galleran H, Pasticier G, et al. Comparative effectiveness of [(18) F]-fluorocholine PET-CT and pelvic MRI with diffusion- weighted imaging for staging in patients with high-risk prostate cancer. Prostate 2015;75:323-331.

17. Ficarra $V$, Novara $G$, Ahlering $T E$, et al. Systematic review and metaanalysis of studies reporting potency rates after robot-assisted radical prostatectomy. Eur Urol 2012;62:418-430.

18. McClure TD, Margolis DJ, Reiter RE, et al. Use of MR imaging to determine preservation of the neurovascular bundles at roboticassisted laparoscopic prostatectomy. Radiology 2012;262:874-883.

19. Rud E, Baco E, Klotz D, et al. Does preoperative magnetic resonance imaging reduce the rate of positive surgical margins at radical prostatectomy in a randomised clinical trial? Eur Urol 2015;68:487-496.

20. Schiavina R, Bianchi L, Borghesi M, et al. MRI displays the prostatic cancer anatomy and improves the bundles management before robot-assisted radical prostatectomy. J Endourol 2018;32:315-321.

21. Radtke JP, Hadaschik BA, Wolf MB, et al. The impact of magnetic resonance imaging on prediction of extraprostatic extension and prostatectomy outcome in patients with low-, intermediateand high-risk prostate cancer: try to find a standard. J Endourol 2015;29:1396-1405.

22. Yossepowitch O, Briganti A, Eastham JA, et al. Positive surgical margins after radical prostatectomy: a systematic review and contemporary update. Eur Urol 2014;65:303-313. 